Original article

\title{
Preoperative chronic opioid use and its impact on early complications in bariatric surgery: a Swedish nationwide cohort study of 56,183 patients
}

\author{
Martin L. Skogar, M.D., Ph.D.* , Magnus Sundbom, M.D., Ph.D. \\ Department of Surgical Sciences, Uppsala University, Uppsala, Sweden \\ Received 11 January 2021; accepted 4 April 2021
}

\begin{abstract}
Background: The association between severe obesity and chronic pain makes opioid use common among bariatric patients. Preoperative opioid use has been identified as a risk factor in other surgical procedures.

Objectives: To examine the impact of preoperative opioid use on complications after primary bariatric surgery.

Setting: Sweden.

Methods: All primary laparoscopic Roux-en-Y gastric bypass (LRYGB) and laparoscopic sleeve gastrectomy (LSG) patients from 2007-2017 were identified in the Scandinavian Obesity Surgery Register. Prescriptions for opioids within 90 days prior to surgery were retrieved from the Swedish Prescribed Drug Register and converted into oral morphine equivalents (OMEs). Patients with $\geq 2$ prescription of opioids within 90 days prior to surgery were defined as chronic opioid users. Generalized linear regression was used to adjust for age, sex, body mass index, procedure type, year of operation, and co-morbidities.

Results: Of the 56,183 patients who had undergone primary LRYGB $(n=49,615)$ or LSG $(n=6568)$, $17.5 \%(n=9825)$ had at least 1 prescription of opioids prior to surgery, of which $4.3 \%(n=2390)$ were defined as chronic opioid users. Chronic opioid use was associated with a higher risk of severe complications (Clavien Dindo grade $\geq 3 \mathrm{~b}$; odds ratio [OR], 1.67; 95\% confidence interval [CI], 1.37-2.04), increased lengths of stay (relative risk, 1.11; 95\% CI, 1.08-1.14), and higher rates of readmission (OR, 1.70; 95\% CI, 1.49-1.94) and reoperation (OR, 1.87; 95\% CI, 1.53-2.27; all $P$ values $<.001)$. Furthermore, higher OME exposure was associated with stepwise higher risks.

Conclusion: Preoperative opioid use was an independent risk factor for severe complications, as well as prolonged lengths of stay, readmission, and reoperation after primary bariatric surgery. (Surg Obes Relat Dis 2021;17:1256-1262.) (C) 2021 American Society for Bariatric Surgery. Published by Elsevier Inc. This is an open access article under the CC BY license (http://creativecommons.org/licenses/ by/4.0/).
\end{abstract}

Keywords: $\quad$ Gastric bypass; Sleeve gastrectomy; Bariatric surgery; Opioid; Chronic pain; Oral morphine equivalents; Complication

Because the prevalence of obesity continues to rise rapidly [1], an increasing number of individuals worldwide face multiple obesity-related diseases and premature death
[2]. In addition to the more specific metabolic diseases, obesity is significantly associated with pain, probably due to biomechanical stress on weight-bearing joints, various

* Correspondence: Martin L Skogar, M.D., Ph.D., Department of Surgical Sciences, Uppsala University,SE-751 85, Uppsala, Sweden. 
inflammatory mediators, and lifestyle issues [3]. The increasing use of opioid analgesics [4], known to increase the risk for opioid-associated adverse effects and mortality [5,6], is also a concern in bariatric patients [7]. Hence, this group of individuals suffers from 2 major health concerns, both of which have reached epidemic proportions $[8,9]$.

Known risk factors for an increased complication risk after laparoscopic Roux-en-Y gastric bypass (LRYGB) and laparoscopic sleeve gastrectomy (LSG) are revisional surgery, older age, high body mass index (BMI), male sex, smoking, and co-morbidities, such as diabetes and pulmonary and cardiovascular diseases [10-12]. Preoperative use of opioids has also been found to be an independent risk factor for increased lengths of stay (LOSs), higher 30-day readmission rates, and higher costs in the postoperative period after various elective abdominal procedures [13,14]. Concerning bariatric surgery, Tian et al. [15] recently reported prolonged LOSs and a higher 30-day complication rate in 5357 patients, while Shockcor et al. [16] reported similar complication rates in 130 chronic opioid users matched with propensity scores to 130 opioid-naïve patients.

The aim of this study was to examine the impacts of preoperative opioid use on complications, lengths of stay, and readmission and reoperation rates within 30 days in a large, nationwide cohort undergoing primary LRYGB and LSG.

\section{Methods}

\section{Setting and population}

Data from the Scandinavian Obesity Surgery Register (SOReg) between 2007 and 2017 were used for this retrospective cohort study. The database captures baseline data (age, sex, obesity-related co-morbidities, date of surgery, and type of procedure) and subsequent follow-up data at 6 weeks and 1, 2, 5, and 10 years. Since its start in 2007, SOReg has included $97.4 \%$ of all bariatric procedures performed in Sweden [17]. All primary LRYGB and LSG cases were included in this study. The study was approved by the Regional Ethical Review Board.

\section{Exposure and outcome}

The exposure, prescribed opioid analgesics, was retrieved from the Swedish Prescribed Drug Register [18], which collect data on all prescribed medications that are dispensed at any Swedish pharmacy. The dispensed opioid analgesic was identified by the Anatomical Therapeutic Classification system (N02A). The outcome variables, complications within 30 days, LOSs, and readmissions and reoperations within 30 days, were obtained from SOReg. Our primary outcome variable, 30-day complications, was dichotomized into cases with and without severe complications. A severe complication was defined as a case with a Clavien Dindo grade of $3 b$ or worse $(C D \geq 3 b)$, indicating a complication requiring intervention under general anesthesia, treatment at an intensive care unit, or death [19].

\section{Preoperative opioid use}

Patients were classified as chronic opioid users if they had at least 1 prescription of opioid analgesics within 30 to 90 days prior to surgery and at least another prescription 30 days prior to surgery, in order to differentiate them from patients with occasional use of opioids. Each opioid prescription was first converted into milligrams and then converted into average daily oral morphine equivalents (OMEs) using the morphine equivalent conversion factor per milligram for the specific opioid [20]. The total average daily OME exposure within 90 days prior to surgery was calculated by adding up OME dosages of all opioid prescriptions within this period and dividing by the total number of days. Hence, 2 independent variables were created describing the use of opioids before surgery: (1) chronic use of an opioid or not (dichotomous); and (2) average daily OME dosage during the 90 days prior to surgery $(\mathrm{mg} / \mathrm{d})$. The latter variable was categorized into 4 groups: (1) opioid naïve and (2) low (>0-10); (3) middle ( $>10-20)$; and (4) high OME exposure $(>20 \mathrm{mg} / \mathrm{d})$.

\section{Covariates}

Information on sex, age, BMI, year of surgery, type of procedure, and the obesity-related co-morbidities of depression, diabetes, dyspepsia, hypertension (dichotomized on medication or not), and sleep apnea (use of continuous positive airway pressure), as well as previous venous thromboembolism (VTE), were obtained from SOReg.

\section{Statistical analysis}

The independent sample $t$ test and Fischer's exact test were used as univariate analyses for continuous and categorical variables, respectively. Continuous variables were reported as means \pm standard deviations and categorical variables as proportions.

Two regression models were constructed for each of the outcome variables, each with 1 of the 2 variables describing use of opioids before surgery. The models were adjusted for differences in age, sex, BMI, procedure type, year of operation, and co-morbidities. All covariates were tested for collinearity. Binary logistic regression was used for 30day complications, 30-day readmissions, and 30-day reoperations, whereas LOS data had a Poisson distribution for which a Poisson regression model was used.

Using the odds ratio (OR) from logistic regression and relative risk (RR) from Poisson regression, adjusted rates of 30-day complications, adjusted mean LOSs, adjusted 30-day readmissions, and adjusted 30-day reoperations were back-calculated. 
All $P$ values were 2 -sided, and a $P$ value $<.05$ was considered statistically significant. All analyses were done using IBM SPSS Statistics version 27.

\section{Results}

In total, 56,183 patients were included in the study, $88.3 \%$ ( $\mathrm{n}=49,615)$ were patients who underwent LRYGB and $11.7 \%(\mathrm{n}=6568) \mathrm{LSG}$, whereof $17.5 \%$ had at least 1 opioid prescription prior to surgery and $4.3 \%$ were chronic opioid users. Chronic opioid users were older and had more coexisting co-morbidities (Table 1). Overall, $82.5 \%(\mathrm{n}=46,358)$ were opioid naïve, while $14.2 \%(\mathrm{n}=7954), 1.7 \%(\mathrm{n}=$ $961)$, and $1.6 \%(\mathrm{n}=909)$ had low $(<10 \mathrm{mg} / \mathrm{d})$, middle $(10-20 \mathrm{mg} / \mathrm{d})$, and high $(>20 \mathrm{mg} / \mathrm{d})$ OME exposure, respectively.

The most prescribed opioid was tramadol $(45.6 \%$ of all prescriptions), followed by codeine (30.5\%), oxycodone $(14.5 \%)$, morphine $(2.7 \%)$, and buprenorphine $(2.3 \%)$.

\section{Severe complication}

In the total cohort of 56,183 patients, the overall 30-day complication rate was $6.9 \%(\mathrm{n}=3868)$, of which $2.8 \%$ $(\mathrm{n}=1547)$ had a severe complication $(\mathrm{CD} \geq 3 \mathrm{~b})$. A severe complication occurred more frequently among all chronic opioid users $(4.7 \%[\mathrm{n}=112]$ versus $2.7 \%[\mathrm{n}=1435] ; P<$ .001 in a univariate analysis). After a multivariate regression analysis, chronic opioid use was still associated with an increased risk of a severe complication (OR, 1.67; 95\% confidence interval $[\mathrm{CI}], 1.37-2.04 ; P<.001$ ), with an adjusted 30-day complication rate of $4.4 \%$ (Table 2). A stepwise relationship between OME exposure and risk for a severe complication was seen (Fig. 1A). In a subanalysis of specific complications, chronic opioid use was associated with increased risks of leakage/abscess (OR, 1.60; 95\% CI, 1.22-2.10), bleeding (OR, 1.93; 95\% CI, 1.542.42), bowel obstruction (OR, 1.72; 95\% CI, 1.22-2.44), and pulmonary complications (OR, 1.56; 95\% CI, 1.032.36; Table 3).

\section{Length of stay}

The mean LOS was $1.9 \pm 2.8$ days overall. Chronic opioid users had a slightly longer LOS compared to the other patients $(2.1 \pm 3.7$ versus $1.9 \pm 2.7 \mathrm{~d}$, respectively; $P<.001$ in a univariate analysis). After the multivariate regression analysis, the RR of an increased LOS remained significantly higher for chronic opioid users (RR, 1.11; 95\% CI, 1.08-1.14; $P<.001$; Table 2). Opioid users had a significantly higher risk for a longer hospital stay, except for those with low OME exposure, compared to the opioid-naïve group (Fig. 1B).

\section{The 30-day readmission rate}

The overall 30-day readmission rate was $7.0 \%(\mathrm{n}=$ 3911). A higher rate was observed among chronic opioid users $(11.3 \%[\mathrm{n}=269]$ versus $6.8 \%[\mathrm{n}=3642] ; P<$ .001 in a univariate analysis). After the multivariate regression analysis, chronic opioid use was still associated with an increased risk of readmission within 30 days (OR, 1.70; 95\% CI, 1.49-1.94; $P<.001$; Table 2). A stepwise relationship between OME exposure and the risk for readmission within 30 days was seen (Fig. 1C).

Table 1

Unadjusted characteristics of individuals with bariatric surgery, with and without chronic opioid use

\begin{tabular}{|c|c|c|c|}
\hline & \multicolumn{2}{|c|}{ Opioid use prior to bariatric surgery } & \multirow[t]{2}{*}{$P$ value } \\
\hline & None or occasional* & Chronic $^{\dagger}$ & \\
\hline Total, n (\%) & $53,793(95.7)$ & $2390(4.3)$ & \\
\hline Sex, female, n $(\%)$ & $41,104(76.4)$ & $1811(75.8)$ & .476 \\
\hline Age, yr, mean $\pm \mathrm{SD}$ & $40.7 \pm 11.2$ & $45.4 \pm 9.8$ & $<.001$ \\
\hline BMI, $\mathrm{kg} / \mathrm{m}^{2}$, mean $\pm \mathrm{SD}$ & $41.9 \pm 5.5$ & $41.4 \pm 5.6$ & $<.001$ \\
\hline \multicolumn{4}{|l|}{ Operation, n (\%) } \\
\hline LRYGB & $47,534(88.4)$ & $2081(87.1)$ & .054 \\
\hline LSG & 6259 (11.6) & $309(12.9)$ & \\
\hline \multicolumn{4}{|c|}{ Co-morbidities (on medication) } \\
\hline Depression, n (\%) & $7745(14.4)$ & $690(28.9)$ & $<.001$ \\
\hline Diabetes, n (\%) & $7158(13.3)$ & $416(17.4)$ & $<.001$ \\
\hline Dyspepsia, n (\%) & $4848(9.0)$ & $442(18.5)$ & $<.001$ \\
\hline Hypertension, n (\%) & $12,943(24.1)$ & 857 (35.9) & $<.001$ \\
\hline Previous VTE, n (\%) & $1136(2.1)$ & $103(4.3)$ & $<.001$ \\
\hline Sleep apnea, n (\%) & $5035(9.4)$ & $294(12.3)$ & $<.001$ \\
\hline
\end{tabular}

$\mathrm{SD}=$ standard deviation; BMI = body mass index; LRYGB = laparoscopic Roux-en-Y gastric bypass; LSG = laparoscopic sleeve gastrectomy; VTE $=$ venous thromboembolism.

* Opioid-naïve patients $(82.5 \% ; \mathrm{n}=46,358)$ and those with occasional use $(13.2 \% ; \mathrm{n}=7435)$.

${ }^{\dagger}$ Chronic opioid use was defined as $\geq 1$ prescription of opioid 30-90 days prior to surgery and $\geq 1$ prescription 0-30 days prior to surgery. 
Table 2

Unadjusted and adjusted differences in healthcare use for chronic opioid users $(\mathrm{n}=2390)$ and the remaining patients $(\mathrm{n}=53,793)$

\begin{tabular}{|c|c|c|c|c|c|c|}
\hline & \multicolumn{6}{|c|}{ Opioid use prior to bariatric surgery } \\
\hline & \multicolumn{3}{|l|}{ Unadjusted } & \multicolumn{3}{|l|}{ Adjusted $^{\ddagger}$} \\
\hline & None or occasional* & Chronic $^{\dagger}$ & $P$ value & Chronic $^{\dagger}$ & OR $(95 \% \mathrm{CI})$ & $P$ value \\
\hline 30-d severe complication, $\%$ & 2.7 & 4.7 & $<.001$ & 4.4 & $1.67(1.37-2.04)$ & $<.001$ \\
\hline Length of stay, days & 1.9 & 2.1 & $<.001$ & 2.1 & $1.11(1.08-1.14)^{\S}$ & $<.001$ \\
\hline 30-d readmission, $\%$ & 6.8 & 11.3 & $<.001$ & 11.0 & $1.70(1.49-1.94)$ & $<.001$ \\
\hline 30-d reoperation, $\%$ & 2.5 & 4.9 & $<.001$ & 4.6 & $1.87(1.53-2.27)$ & $<.001$ \\
\hline
\end{tabular}

$\mathrm{OR}=$ odds ratio $; \mathrm{CI}=$ confidence interval.

* Opioid-naïve patients $(82.5 \% ; \mathrm{n}=46,358)$ and those with occasional use $(13.2 \% ; \mathrm{n}=7435)$.

${ }^{\dagger}$ Chronic opioid use was defined as $\geq 1$ prescription of opioids 30-90 days prior to surgery and $\geq 1$ prescription $0-30$ days prior to surgery.

$\ddagger$ Adjusted for differences in baseline characteristics (sex, age, body mass index, year of surgery, procedure type, and co-morbidities).

$\S$ Relative risk.

\section{The 30-day reoperation rate}

Overall, 2.6\% $(\mathrm{n}=1480)$ had a reoperation related to a complication within 30 days, which was more common among chronic opioid users $(4.9 \%[\mathrm{n}=117]$ versus $2.5 \%[\mathrm{n}=1363] ; P<.001$ in a univariate analysis). After

A
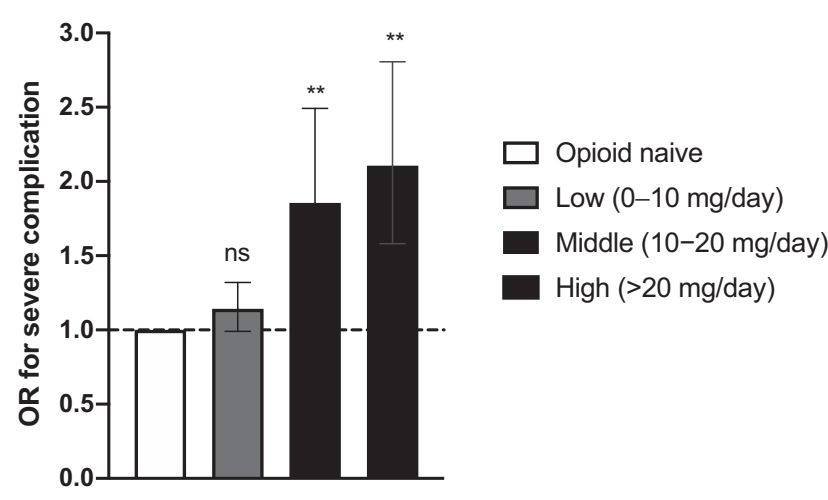

C

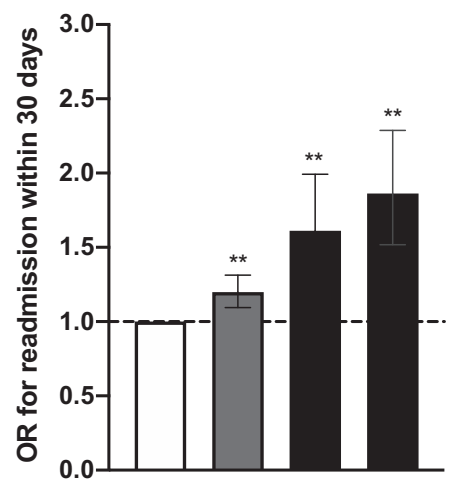

the multivariate regression analysis, chronic opioid use was still associated with an increased risk for reoperation within 30 days (OR, 1.87; 95\% CI, 1.53-2.27; $P<.001$; Table 2). A stepwise relationship between OME exposure and the risk for reoperation within 30 days was seen (Fig. 1D).

B



D

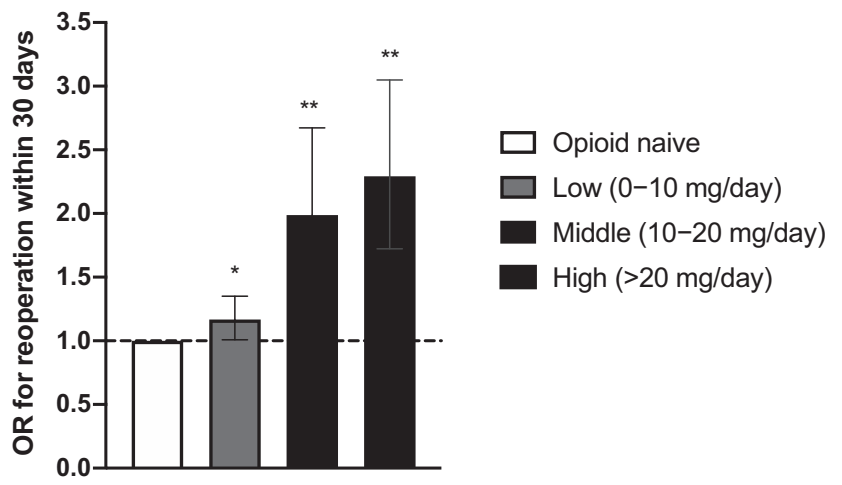

Fig. 1. ORs or RRs with $95 \%$ CIs for (A) severe complications (Clavien Dindo grade $\geq 3$ b; i.e., intervention in general anesthesia or treatment at an intensive care unit); (B) prolonged hospital stays; (C) 30-day readmissions; and (D) 30-day reoperations in the 4 groups with increasing average daily OMEs. All analyses were adjusted for differences in baseline characteristics (sex, age, BMI, year of surgery, procedure type, and co-morbidities). $* P<.05$; $* * P<.001$. OR $=$ odds ratio; $\mathrm{RR}=$ relative risk; $\mathrm{OME}=$ oral morphine equivalents; $\mathrm{BMI}=$ body mass index; $\mathrm{ns}=$ nonsignificant. 
Table 3

Complications within 30 days for chronic opioid users $(n=2390)$ and the remaining patients $(n=53,793)$

\begin{tabular}{llllll}
\hline & \multicolumn{2}{l}{ Opioid use prior to bariatric surgery } & \multirow{2}{*}{ OR $(95 \% \text { CI })^{\S}$} & $P$ value $^{\S}$ \\
\cline { 2 - 4 } & None or occasional ${ }^{*}$ & Chronic $^{\dagger}$ & $P$ value $^{\ddagger}$ & & \\
\hline Leakage/abscess, n (\%) & $748(1.4)$ & $60(2.5)$ & $<.001$ & $1.60(1.22-2.10)$ & .001 \\
Bleeding, n (\%) & $925(1.7)$ & $88(3.7)$ & $<.001$ & $1.93(1.54-2.42)$ & $<.001$ \\
Bowel obstruction, n (\%) & $495(.9)$ & $36(1.5)$ & .005 & $1.72(1.22-2.44)$ & .002 \\
Cardiovascular event, n (\%) & $76(.1)$ & $5(.2)$ & .398 & $1.04(.42-2.58)$ & .941 \\
Thromboembolism, n (\%) & $48(.1)$ & $2(.1)$ & .999 & $.72(.17-2.98)$ & .645 \\
Pulmonary complication, n (\%) & $312(.6)$ & $25(1.0)$ & .009 & $1.56(1.03-2.36)$ & .036 \\
\hline
\end{tabular}

$\mathrm{OR}=$ odds ratio $\mathrm{CI}=$ confidence interval.

More than 1 complication was possible for each patient.

* Opioid-naïve patients $(82.5 \% ; \mathrm{n}=46,358)$ and those with occasional use (13.2\%; $\mathrm{n}=7435)$.

${ }^{\dagger}$ Chronic opioid use was defined as $\geq 1$ prescription of opioids $30-90$ days prior to surgery and $\geq 1$ prescription $0-30$ days prior to surgery.

${ }^{\ddagger}$ Unadjusted analysis.

${ }^{\S}$ Adjusted for differences in baseline characteristics (sex, age, body mass index, year of surgery, procedure type, and co-morbidities).

\section{Subgroup analysis of LRYGB and LSG}

A subgroup analysis of patients who underwent LRYGB $(\mathrm{n}=49,615)$ and LSG $(\mathrm{n}=6568)$ was performed. In the multivariate regression analysis, chronic opioid users in the LRYGB group had increased risks of a severe complication (OR, 1.74; 95\% CI, 1.42-2.14; $P<.001$ ), a longer LOS (RR, 1.12; 95\% CI, 1.08-1.15; $P<.001$ ), readmission within 30 days (OR, 1.73; 95\% CI, 1.51-1.99; $P<.001$ ), and reoperation within 30 days (OR, 1.86; 95\% CI, 1.542.25; $P<.001)$. No such associations were observed in the LSG group alone (severe complication OR, .85 [95\% CI, .34-2.13; $P=.732$ ]; LOS OR, 1.07 [95\% CI, .98$1.17 ; P=.118$ ]; 30-d readmission OR, 1.33 [95\% CI, $.81-2.17 ; P=.263$ ]; 30-d reoperation OR, .84 [95\% CI, $.34-2.10 ; P=.841])$.

\section{Discussion}

In this cohort of 56,183 patients who underwent primary laparoscopic bariatric surgery (gastric bypass or sleeve gastrectomy), $17.5 \%$ of the patients had at least 1 prescription of opioids prior to surgery, of which $4.3 \%$ were defined as chronic opioid users. The latter group of patients had an increased risk of severe complications (OR, 1.67; 95\% CI, 1.37-2.04), as well as prolonged LOSs (RR, 1.11; 95\% CI, 1.08-1.14) and higher rates of both readmissions (OR, 1.70; 95\% CI, 1.49-1.94) and reoperations (OR, 1.78; 95\% CI, 1.48-2.14; all $P$ values $<.001)$ within the first 30 postoperative days. In addition, a stepwise positive correlation was observed between total average OME exposure and an increased risk for an adverse outcome (severe complication, longer LOS, and higher 30-day readmission and reoperation rates).

The prevalence of opioid users in this cohort of bariatric patients was remarkably high $(17.5 \%$ with $\geq 1$ dispensed opioid within 90 days) compared to the Swedish population in general $(6 \%-8 \%$ with $\geq 1$ dispensed opioid during a calendar year) [21,22], which highlights the association between severe obesity and pain. As an international comparison, the prevalence of opioid use before bariatric surgery has been reported to be $14.7 \%$ in the United States [23] and $12 \%$ in Canada [15]. The slightly higher rate observed in this study might be explained by our access to the Swedish Prescribed Drug Register, which made it possible to identify all dispensed opioid prescriptions, whereas the 2 other studies captured opioid use by patient reports, chart reviews from the preoperative assessment, or both. In comparison, $8 \%$ of patients were identified as chronic opioid users before bariatric surgery (defined as $\geq 10$ opioids dispensed over $\geq 90 \mathrm{~d}$ or a $\geq 120$-d supply of opioids dispensed during the year before surgery) in the United States [7], compared with $4.3 \%$ in our study; however, the definitions of chronic opioid use differed. Pain lasting more than 3 months is often referred to as chronic pain [24]; hence, our definition of chronic opioid use was based on repetitive prescriptions within 90 days prior to surgery. More than $75 \%$ of prescribed opioids in our study were "weak opioids" (Codeine, Tramadol), while higher doses and stronger opioids seem to be more commonly prescribed in North America [25].

The negative impact of preoperative opioid use has previously been reported in spine surgery (increased LOS) [26], orthopedic surgery (increased postoperative morbidity, mortality, and resource utilization) [27], general abdominal surgery (higher costs, increased LOSs, more complications, and readmissions) $[13,14]$, and bariatric surgery (prolonged LOSs and higher 30-d complication rate) [15]. However, conflicting results have been reported in bariatric surgery [16]. The present study, consisting of a large, nationwide cohort with cross-matching data from 2 national registers with high coverage, establishes the increased risks and enables a better understanding of the relationship between higher opioid dosages and risks of complications after bariatric surgery. In the subgroup analysis of LRYGB and LSG, a worsened outcome was observed only in the LRYGB 
group; therefore, we agree with Tian et al. [15], that LSG might be a safer choice in patients using opioids preoperatively.

In clinical practice, bariatric surgeons and staff might become insecure when evaluating postoperative patients with ongoing opioid use, because these individuals could present an abnormally high level of pain due to undertreatment of their underlying pain-related condition or having a lower pain threshold. Maintaining an increased level of safety could therefore lead to a diagnostic laparoscopy (graded as a severe complication) or an extended observation time (increased LOS), as well as increased risk for readmission, reoperation, or both if the patient returns within the first 30 days. Respiratory depression, impaired bowel movement, and opioid-induced hyperalgesia, 3 well-known adverse effects of opioids, might also play an important role in the longer LOSs and higher rates of early readmissions observed in this study. Interestingly, postoperative bowel obstruction was more often observed for patients with chronic opioid use (1.5\% versus .9\%). Moreover, the immunosuppressive effects of opioids are likely another important factor. Increasing dosages and prolonged treatment have been found to have additive impairments on the innate and adaptive immune system [28]. In a clinical study, opioid use was found to be associated with increased risks of infections and pneumonia [29]. An intact immune system has a central role in tissue healing after surgery, and an impaired immune system might therefore lead to an increased risk of a severe complication and the need for reoperation. Based on these findings, it seems likely that chronic opioid users, particularly those with high dosages, may be more susceptible to complications related to poorer tissue healing and infections [30].

Although this study focuses on preoperative opioid use, correct and safe perioperative use is probably of greater concern. Recently, an international multidisciplinary consensus panel developed strategies to prevent opioidrelated harm in adult surgical patients postoperatively [31]. Among these, a preoperative assessment of the risk of persistent postoperative opioid use, avoidance of longacting opioid formulations, limiting the number of tablets prescribed at discharge, and avoidance of automatic prescription refills, as well as better education of healthcare professionals and patients, are mentioned. Previous studies have reported increased use of opioids after bariatric surgery [7,32]. The association between opioid use preoperatively and the increased risk of an adverse outcome after bariatric surgery, as observed in this study, may at least partly be a contributing factor.

\section{Limitations}

This registry-based study has several inherent limitations. Firstly, the indication for the prescription of opioids was unknown, and there were potential differences between dispensed prescriptions of opioids and actual use. Musculoskeletal pain was observed in $21 \%(\mathrm{n}=512)$ of our chronic opioid users in SOReg; however, as this is a nonmandatory variable, data were missing in 58.2\%. Moreover, our decision to compare chronic opioid users to the remaining patients (opioid naïve and those with occasional use) may underestimate the effects of chronic opioid use. Secondly, the presence of co-morbidities was binary and was defined based on whether a patient used ongoing medication. Thirdly, some residual confounding factors may exist, such as former abdominal surgery and specific pain disorders, for which we were not able to adjust. However, the results were in line with previous studies, and a clear stepwise increase was observed in our outcome variables.

\section{Conclusion}

Preoperative opioid use is common among patients undergoing bariatric surgery and represents an independent risk factor for severe complications, as well as prolonged lengths of stay, readmission, and reoperation after primary LRYGB and LSG. Furthermore, higher OME exposure was associated with stepwise higher risks. Patients using opioid analgesics before bariatric surgery require special attention during the perioperative phase.

\section{Disclosures}

The authors have no commercial associations that might be a conflict of interest in relation to this article.

\section{References}

[1] NCD Risk Factor Collaboration. Trends in adult body-mass index in 200 countries from 1975 to 2014: a pooled analysis of 1698 population-based measurement studies with 19.2 million participants. Lancet 2016;387(10026):1377-96.

[2] Flegal KM, Kit BK, Orpana H, Graubard BI. Association of all-cause mortality with overweight and obesity using standard body mass index categories: a systematic review and meta-analysis. JAMA 2013;309(1):71-82.

[3] Okifuji A, Hare BD. The association between chronic pain and obesity. J Pain Res 2015;8:399-408.

[4] Gomes T, Juurlink DN, Dhalla IA, Mailis-Gagnon A, Paterson JM, Mamdani MM. Trends in opioid use and dosing among socioeconomically disadvantaged patients. Open Med 2011;5(1):e13-22.

[5] Benyamin R, Trescot AM, Datta S, et al. Opioid complications and side effects. Pain Physician 2008;11(Suppl 2):S105-20.

[6] Fischer B, Rehm J. Deaths related to the use of prescription opioids. CMAJ 2009;181(12):881-2.

[7] Raebel MA, Newcomer SR, Reifler LM, et al. Chronic use of opioid medications before and after bariatric surgery. JAMA 2013;310(13):1369-76.

[8] Jaacks LM, Vandevijvere S, Pan A, et al. The obesity transition: stages of the global epidemic. Lancet Diabetes Endocrinol 2019;7(3):23140.

[9] Skolnick P. The opioid epidemic: crisis and solutions. Annu Rev Pharmacol Toxicol 2018;58:143-59.

[10] Maciejewski ML, Winegar DA, Farley JF, Wolfe BM, DeMaria EJ. Risk stratification of serious adverse events after gastric bypass in 
the Bariatric Outcomes Longitudinal Database. Surg Obes Relat Dis 2012;8(6):671-7.

[11] Finks JF, Kole KL, Yenumula PR, et al. Predicting risk for serious complications with bariatric surgery: results from the Michigan Bariatric Surgery Collaborative. Ann Surg 2011;254(4):633-40.

[12] Stenberg E, Cao Y, Szabo E, Naslund E, Naslund I, Ottosson J. Risk prediction model for severe postoperative complication in bariatric surgery. Obes Surg 2018;28(7):1869-75.

[13] Waljee JF, Cron DC, Steiger RM, Zhong L, Englesbe MJ, Brummett CM. Effect of preoperative opioid exposure on healthcare utilization and expenditures following elective abdominal surgery. Ann Surg 2017;265(4):715-21.

[14] Cron DC, Englesbe MJ, Bolton CJ, et al. Preoperative opioid use is independently associated with increased costs and worse outcomes after major abdominal surgery. Ann Surg 2017;265(4):695-701.

[15] Tian C, Maeda A, Okrainec A, Anvari M, Jackson T. Impact of preoperative opioid use on health outcomes after bariatric surgery. Surg Obes Relat Dis 2020;16(6):768-76.

[16] Shockcor N, Adnan SM, Siegel A, Tannouri S, Rasko Y, Kligman M. Preoperative opioid prescription patients do not suffer distinct outcomes after bariatric surgery: a matched analysis of outcomes. Obes Surg 2020;30(10):4014-8.

[17] Sundbom M, Näslund I, Näslund E, Ottosson J. High acquisition rate and internal validity in the Scandinavian Obesity Surgery Registry. Surg Obes Relat Dis 2021;17(3):606-14.

[18] Wallerstedt SM, Wettermark B, Hoffmann M. The first decade with the Swedish Prescribed Drug Register-a systematic review of the output in the scientific literature. Basic Clin Pharmacol Toxicol 2016;119(5):464-9.

[19] Dindo D, Demartines N, Clavien PA. Classification of surgical complications: a new proposal with evaluation in a cohort of 6336 patients and results of a survey. Ann Surg 2004;240(2):205-13.

[20] Nielsen S, Degenhardt L, Hoban B, Gisev N. A synthesis of oral morphine equivalents (OME) for opioid utilisation studies. Pharmacoepidemiol Drug Saf 2016;25(6):733-7.
[21] Jarlbaek L. Opioid prescribing habits differ between Denmark, Sweden and Norway-and they change over time. Scand J Pain 2019;19(3):491-9.

[22] Muller AE, Clausen T, Sjogren P, Odsbu I, Skurtveit S. Prescribed opioid analgesic use developments in three Nordic countries, 20062017. Scand J Pain 2019;19(2):345-53.

[23] King WC, Chen JY, Belle SH, et al. Use of prescribed opioids before and after bariatric surgery: prospective evidence from a U.S. multicenter cohort study. Surg Obes Relat Dis 2017;13(8):1337-46.

[24] Dowell D, Haegerich TM, Chou R. CDC guideline for prescribing opioids for chronic pain-United States, 2016. MMWR Recomm Rep 2016;65(1):1-49.

[25] Ladha KS, Neuman MD, Broms G, et al. Opioid prescribing after surgery in the United States, Canada, and Sweden. JAMA Netw Open 2019;2(9):e1910734.

[26] Armaghani SJ, Lee DS, Bible JE, et al. Increased preoperative narcotic use and its association with postoperative complications and length of hospital stay in patients undergoing spine surgery. Clin Spine Surg 2016;29(2):E93-8.

[27] Menendez ME, Ring D, Bateman BT. Preoperative opioid misuse is associated with increased morbidity and mortality after elective orthopaedic surgery. Clin Orthop Relat Res 2015;473(7):2402-12.

[28] Plein LM, Rittner HL. Opioids and the immune system-friend or foe. Br J Pharmacol 2018;175(14):2717-25.

[29] Wiese AD, Griffin MR, Schaffner W, et al. Opioid analgesic use and risk for invasive pneumococcal diseases: a nested case-control study. Ann Intern Med 2018;168(6):396-404.

[30] Moyano J, Aguirre L. Opioids in the immune system: from experimental studies to clinical practice. Rev Assoc Med Bras (1992) 2019;65(2):262-9.

[31] Levy N, Quinlan J, El-Boghdadly K, et al. An international multidisciplinary consensus statement on the prevention of opioid-related harm in adult surgical patients. Anaesthesia 2021;76(4):520-36.

[32] Wallen S, Szabo E, Palmetun-Ekback M, Naslund I. Use of opioid analgesics before and after gastric bypass surgery in Sweden: a population-based study. Obes Surg 2018;28(11):3518-23. 